which often lack sufficient sensitivity and specificity for the accurate diagnosis needed to determine prognosis and guide management. Until recently, surgical biopsy or resection was the only means of obtaining a definitive diagnosis of cystic pancreatic tumors.

Endoscopic ultrasound-guided needle biopsy, using a Tru-cut ${ }^{\circledR}$ (Allegiance Corp., Delaware, USA) needle (EUS TCB), has the potential to sample tissue from cystic pancreatic tumors without the need for surgery. Levy and colleagues aimed to determine whether material obtained by this means was sufficient for histologic diagnosis in a small study $(n=10)$ of patients with cystic pancreatic lesions. They found that EUS TCB was not diagnostic in three of the patients. For the remaining seven cases, however, TCB results guided patient management. EUS TCB obviated the need for surgery in four cases of serous cystadenoma, ruling out metastatic disease in one of these and so guiding radiation therapy, and confirmed the need for surgery in three patients. These results were compared with fine-needle aspiration samples, also taken at the time, which were nondiagnostic in nine patients.

The authors conclude that EUS TCB is safe in patients with cystic pancreatic tumors, and has the potential to yield sufficient tissue for histologic diagnosis. They recommend that a clearer understanding of the use and safety of EUS TCB in cystic pancreatic tumors needs to be achieved by prospective controlled trials in a larger patient group.

Carol Lovegrove

Original article Levy MJ et al. (2005) Endoscopic ultrasound-guided trucut biopsy of the cyst wall for diagnosing cystic pancreatic tumors. Clin Gastroenterol Hepatol 3: 974-979

\section{Clinical manifestations and treatment of food-cobalamin malabsorption in the elderly}

Research has shown that cobalamin (vitamin $B_{12}$ ) deficiency is common in individuals over 60 years of age and is often the result of malabsorption, due to an inability to release the vitamin either from its serum binding protein or from food; however, studies have yet to address food-cobalamin malabsorbtion in the elderly or assess its clinical consequences.
Andrès and colleagues performed an observational cohort study of 92 patients over 65 years of age, with cobalamin deficiency (serum cobalamin levels $<200 \mathrm{pg} / \mathrm{ml}$ ) resulting from food-cobalamin malabsorbtion, by collecting data retrospectively from medical records. Neurologic and psychologic manifestations of the deficiency were found to occur most frequently, with mild sensory polyneuropathy occurring in 41 patients and confusion or impaired mental functioning in 21 patients, respectively. In addition, more than two-thirds of patients had mild hematologic abnormalities, and at least a third of patients had severe hematologic manifestations such as anemia, leukopenia, thrombocytopenia or pancytopenia. At least one associated condition was present in 56 of the patients, of whom 28 showed evidence of atrophic gastritis. In all patients, treatment with intramuscular or oral cyanocobalamin corrected serum cobalamin levels to above $200 \mathrm{pg} / \mathrm{ml}$ and improved hematologic abnormalities.

The authors conclude that, in accordance with previous studies, food-cobalamin malabsorption might be the primary cause of cobalamin deficiency in elderly patients. They stress that larger studies are required to confirm this and also the association between food-cobalamin malabsorption and neurologic, psychologic and hematologic abnormalities.

Katy Cherry

Original article Andrès E et al. (2005) Food-cobalamin malabsorption in elderly patients: clinical manifestations and treatment. Am J Med 118: 1154-1159

\section{Small gallstones increase the risk of acute pancreatitis}

Studies have established a causal role for biliary sludge in pancreatitis and have also suggested that smaller gallstones might also increase the risk; however, this link is still controversial. The association between gallstone size and number and pancreatitis has been investigated retrospectively by Venneman and colleagues, who also evaluated the benefit of two management strategies: the 'wait and see' approach, and prophylactic cholecystectomy.

Patients who underwent cholecystectomy or endoscopic retrograde cholangiopancreatography (ERCP) at the University Medical Center of Utrecht were identified 